\title{
Texture Driven Hierarchical Fusion for Multi-Biometric System
}

\author{
Devendra Reddy Rachapalli ${ }^{1 *}$, Hemantha Kumar Kalluri ${ }^{2}$ \\ ${ }^{1}$ SriKalahasteeswara Institute of Technology, India \\ ${ }^{2}$ Vignan's Foundation for Science, Technology \& Research University, India \\ *Corresponding author E-mail: csdreddy@gmail.com
}

\begin{abstract}
This article presents hierarchical fusion models for multi-biometric systems with improved recognition rate. Isolated texture regions are used to encode spatial variations from the composite biometric image which is generated by signal level fusion scheme. In this paper, the prominent issues of the existing multi-biometric system, namely, fusion methodology, storage complexity, reliability and template security are discussed. Here wavelet decomposition driven multi-resolution approach is used to generate the composite images. Texture feature metrics are extracted from multi-level texture regions and principal component analyzes are evaluated to select potentially useful texture values during template creation. Here through consistency and correlation driven hierarchical feature selection both inter-class similarity and intra-class variance problems can be solved. Finally, t-normalized feature level fusion is incorporated as a last stage to create the most reliable template for the identification process. To ensure the security and add robustness to spoof attacks random key driven permutations are used to encrypt the generated multi-biometric templates before storing it in a database. Our experimental results proved that the proposed hierarchical fusion and feature selection approach can embed fine detailed information about the input multi modal biometric images with the least complex identification process.
\end{abstract}

Keywords: LBP descriptor; multi-biometric; spatial information; texture pattern; wavelet fusion.

\section{Introduction}

In recent years the biometric system has emerged as a basic modality in many real time applications that need authentication and identification tasks [2]. Due to the frequent security breaches that arise with other security systems biometrics is increasingly becoming popular and it has been investigated in several works for improved system performance. Though biometric systems are widely accepted as stand-alone methods still they suffer from some undesirable quality degradations in terms of accuracy and acceptability. In general, feature values are used to explore the characteristics of biometric images [4],[5] which should be precise representations of uniqueness which belongs to each biometric model and also need to be stable for a long time. Considering the variations that may occur in biometric images due to the illumination and scale changes it is necessary to develop multi-modal biometric images which incorporate several unique characteristics of each class to improve the recognition accuracy. Multi-modals have several metrics compared to the uni-modal biometric system since it can able to solve the problems related to uni-modal systems. And it can also reduce the Failure-to-Enroll Rate (FTER) and Failure-to-Capture Rate (FTCR) and ensure the most reliable identification task.

\section{Related works}

Multi-biometric systems [1] are used to improve the reliability through multiple sources of information. And it has following advantages over conventional biometrics systems such as it is a computationally efficient model during the identification process. It will improve the throughput rate. The inherent limitations of the biometric sources can be mitigated with sufficient discrimination during decision-making. It is more resistant to spoofing attacks since multiple biometrics from several sources are used. The major limitations that arise in any multi-biometric systems are increased computational complexity and storage space than unibiometric systems. Biometric fusion methodologies widely preferred to optimize the storage and most reliable identification process. It has been investigated in many existing works and it was proved that there is a substantial impact on the overall system performance by considering the performance metrics of the fusion level. In [3] the signal level fusion operation is performed to combine the iris and face images and the scores are assigned to formulate the composite images and finally normalized using a min-max rule to optimize the modality.

In [6] Log-Gabor transformations driven feature level coding for template creations and particle swarm optimization (PSO) is used for feature selection to minimize the redundant information during template creation. The same approach is extended to find the optimal feature set from multi-modalities [7]. After signal level fusion performed on face-iris multi-modal images the matching scores generated from individual biometric traits form a 2D feature vector. Here area under curve (AUC) is used as fitness evaluation function in PSO feature selection to retain the most relevant feature set to improve the system performance.

In [8] investigated multi-modal system related to iris and fingerprints. The performance metrics of the matching and decision level scoring is evaluated based on different methodologies such as classic sum, weighted sum and fuzzy logic rules to improve the reliability and weighted function is used to give more priority to iris biometric during identification process due to its consistent performance.

In [9] performed feature level fusion on Scale Invariant Feature Transform (SIFT) descriptors extracted from iris and ear biomet- 
rics and Euclidean distance driven matching is used for classification. It is extended to the score and rank-level based fusion scheme [10] to incorporate the quality of the face and iris biometrics samples. In [11] feature level fusion technique is developed that incorporates the feature values from multi-modal biometric traits and forward into the correlation analysis which makes use of discriminant correlation analysis (DCA). This discriminant feature selection process is used to mitigate the correlative problems between-classes and high variance problems associated with in the classes.

Although the proposed work mainly focuses on the extraction of optimal texture parameter followed by feature level fusion process to generate templates which are stored in the database for the identification process. Computation methods and the way the feature values are extracted to explore the finest characteristics of biometrics traits is a more intuitive process since several methods such as statistical measures, spectral features are having inadequate information handling capacity and dimensionality related issues which leads storage complexity. Among the various approaches investigated for feature generation texture driven hierarchical fusion meets the demands required for accurate feature extraction process For merging the associated texture information belongs to various biometrics images not requiring unified infrastructure. Large classes of feature level fusion methodologies are available for compact template generation, offer depth information handling capacity.

In recent years, texture models have gained momentum in biometric applications for high accuracy. The texture metrics based on GLCM (Gray Level Co-occurrence Matrix) are derived from the input biometric images to create the feature set. The GLC matrix is computed over the texture classified image and texture attributes of fused images are forwarded into correlative measures that can be used for finite retention of authentication rate during the matching process.

In this paper, texture information using the template level fusion model are combined to exploit fine details about input biometric image as shown in Fig. 1 which inherent following advantages:

1. Finite discriminative texture model is used to incorporate the spatial details from input biometrics images.
2. It can be easily scaled down using correlative and consistency measures.

3. Hierarchical matching can reduce Failure-to-Enroll Rate (FTER) with improved recognition rate.

In addition, to suppress distortions level during the identification process proposed model includes optimal feature selection for complete robustness to dynamic variations during a biometric scanning process.

\section{Multi-biometric combining process}

\subsection{Fusion model}

In general the image level fusion is widely accepted not only for reducing the storage complexities and also generates more reliable composite images from multiple images to provide better understanding about prominent details. To combine the various classes of biometric models to get precise template value which results with relatively improved identification rate in multi-biometric identification technology. As shown in Fig. 2 only the bands which explore the prominent details are potentially salient and contributing the end results are constructed from spatial variations. Specifically, we select an appropriate sub band according to the desired geometric and texture data retention level. The fusion is performed on the transformed domain and then the inverse transformation is applied to reconstruct the composite image which restores the spatial relationship and intensity values corresponding to both the input biometrics. The input biometric images are down sampled using wavelet transform both in the vertical and horizontal directions. By applying the same process recursively on each decomposed frequency sub bands multi-scale wavelet coefficients are generated. This multi-resolution fusion algorithm uses two different classes of biometric images to generate a single compound fused image. Finally, Root-mean-square error (RMSE) measure as given in (1) is used as an evaluation parameter to validate the efficiency of the proposed fusion model which can accelerate the authentication process with high accuracy.

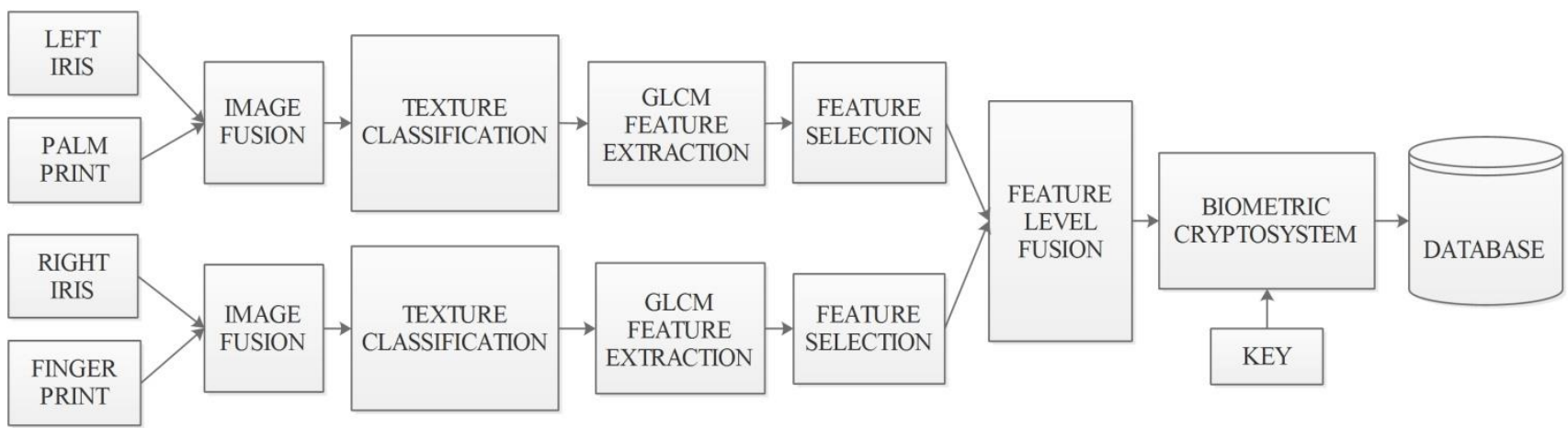

Fig. 1: Multi-biometric template fusion system architecture
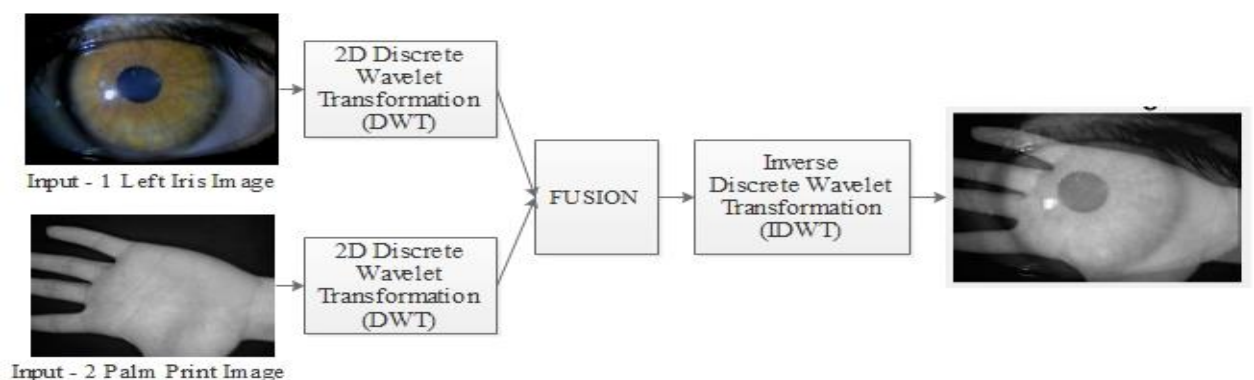

Fig. 2: Wavelet fusion model 


$$
R M S E=\sqrt{\frac{1}{X Y} \sum_{i=1}^{X} \sum_{j=1}^{Y}(I(i, j)-F(i, j))^{2}}
$$

Where, $I(i, j)$ and $F(i, j)$ are input and fused biometric images respectively and the symbols $\mathrm{X}$ and $\mathrm{Y}$ are image sizes.

The CORR measures the similarity between input and the composite biometric images as shown in (2). Its value ranges between -1 and +1 . The +1 represents the highest similarity and -1 for dissimilar.

$C O R R=\frac{2 C_{I F}}{C_{I}+C_{F}}$

Where, $C_{I F}=\sum_{i=1}^{X} \sum_{j=1}^{Y} I(i, j) . F(i, j)$

$$
C_{I}=\sum_{i=1}^{X} \sum_{j=1}^{Y} I(i, j)^{2} \quad C_{F}=\sum_{i=1}^{X} \sum_{j=1}^{Y} F(i, j)^{2}
$$

The proposed model outperformed the state-of-the-art wavelet based hierarchical fusion model which consists of pixel level, feature level and decision level fusions as observed in Table 1.

Table 1: Fusion performance over biometric images

\begin{tabular}{|c|c|c|}
\hline Source Model Used & $\begin{array}{c}\text { Root Mean Square } \\
\text { Error (RMSE) }\end{array}$ & $\begin{array}{c}\text { The correlation coef- } \\
\text { ficient (CORR) }\end{array}$ \\
\hline $\begin{array}{c}\text { Wavelet Fusion - Multi- } \\
\text { Level Approach [12] }\end{array}$ & 5.3566 & 0.8529 \\
\hline Proposed Model & 4.35 & 0.924 \\
\hline
\end{tabular}

\subsection{Rotational invariant texture classification model}

The local binary pattern operator has been used as a standard texture classification approach for texture analysis in many image processing applications. As shown in Fig. 3 the threshold driven Rotational invariant texture models are consistent to the original LBP operator. The basic mathematical operations involved in LBP operation is described in accompanying (3), (4). a)

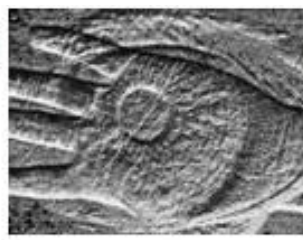

c)

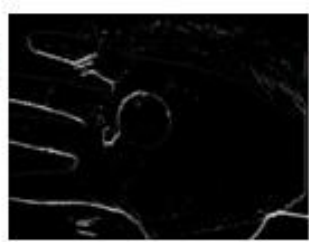

e)

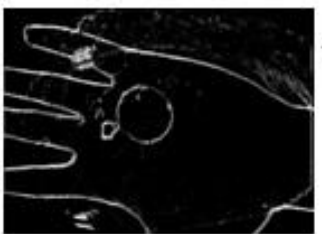

b)

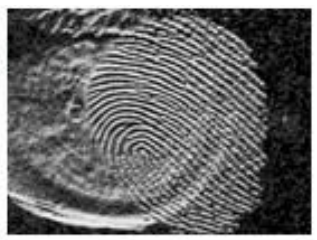

d)
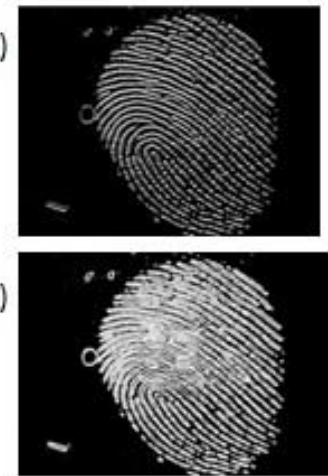

Fig. 3: Binary and ternary description output, binary pattern a) for left iris + palm print $b$ ) for right iris + finger print, ternary pattern c) with upper limit for left iris + palm print d) with upper limit for right iris + finger print, ternary pattern e) with lower limit for left iris + palm print f) with lower limit for right iris + finger print
$L B P_{\text {pixel_out }}=\left\{\sum_{m=0}^{7} 2^{m} * P(G n-G c)\right.$

Where, $G n=$ Neighbour_pixel_value

$G c=$ Central_pixel_value

$P($ value $)=\left\{\begin{array}{l}0, \text { value }<0 \\ 1, \text { value } \geq 0\end{array}\right.$

The LBP patterns address the patterns those have limited transition or discontinuities in binary LBP pattern. Generally, texture binary pattern consists of minimum texture retention since the binary pattern is considered circular. For dynamic textures, texture patterns are depend on threshold margin which is the key element in ternary patterns as shown in (5), where local features have been obtaining with an ability to describe the most discriminative patterns for the multi-biometric template modeling. For ternary pattern upper limit the interested regions are 1 as pixel values and for ternary pattern lower limit the interested regions are -1 as pixel values.

Local Ternary Pattern - lower and upper limits

$N P=\left\{\begin{array}{cc}1, & N P>C P+\text { Thre shold } \\ 0, N P>C P-\text { Thre shold \& \&NP }<C P+\text { Thre shold } \\ -1, & N P<C P-\text { Thre shold }\end{array}\right.$

Where, $N P=$ Neighbour_pixel_value

$C P=C e n t r a l \_p i x e l \_v a l u e$

\subsection{GLCM}

There are different ways to exploit the texture details of an input image in terms of numerical vectors which includes structural, spectral and statistical approaches. Among various statistical approaches which give the detailed description about a spatial relationship, GLCM proved to be the prominent used in many applications. Here GLCM is used to extract texture features which are used to represents a spatial relationship between two biometric traits fused. In general, a texture separation is made by the second order statistical tests with GLCM. It is computed with two parameters such as displacement (d) and orientation $(\varphi)$ between surrounding pixels. Here orientation measures are used with normalization factor which is quantized in multi-direction which includes $0^{\circ}, 45^{\circ}, 90^{\circ}$, and $135^{\circ}$ and computed statistically as shown in (6),(7),(8),(9).

$$
\begin{aligned}
& \text { Correlatia }=\sum_{i, j=0}^{N-1} p_{i j} \frac{(i-\mu)(j-\mu)}{\sigma^{2}} \\
& \text { Energy }=\sum_{i, j=0}^{N-1}\left(p_{i j}\right)^{2}
\end{aligned}
$$

Homogenity $=\sum_{i, j=0}^{N-1} \frac{P_{i j}}{1+(i-j)^{2}}$

Contrast $=\sum_{i, j=0}^{N-1}-\ln \left(P_{i j}\right) P_{i j}$ 


\subsection{Optimal feature selection}

Here Feature selection approaches help to reduce the size of a template without causing severe degradation in performance metrics. Variance measure based PCA transform is used to retain potentially useful texture feature vectors based on redundant and discriminations. Finally, dimensionality reduction is formulated to generate the interested subset's features from a GLCM extracted feature set. The proposed prominent subset selection algorithm includes the following process: consistency based feature normalization to reduce the intra class variance problems associated with multi-modal biometrics, correlation driven subset reduction to mitigate interclass similarity problems.

\subsubsection{Consistency measure for feature normalization}

The consistency measure mainly focused to evaluate the distance of a directional texture feature from the consistency state since texture models are sensitive to the illumination and scale variations. Using the notations introduced above, if the distance value is smaller GLCM features are identical. Here Effective selection is made between binary and ternary texture patterns of two different composite fused images as shown in Fig. 4. The variance level noticed in the biometric sets of an object is called as intra-class variations. The final subset texture attributes belong to one classes need to have high discrimination with lesser in dimension, and should be insensitivity to spatial variations.

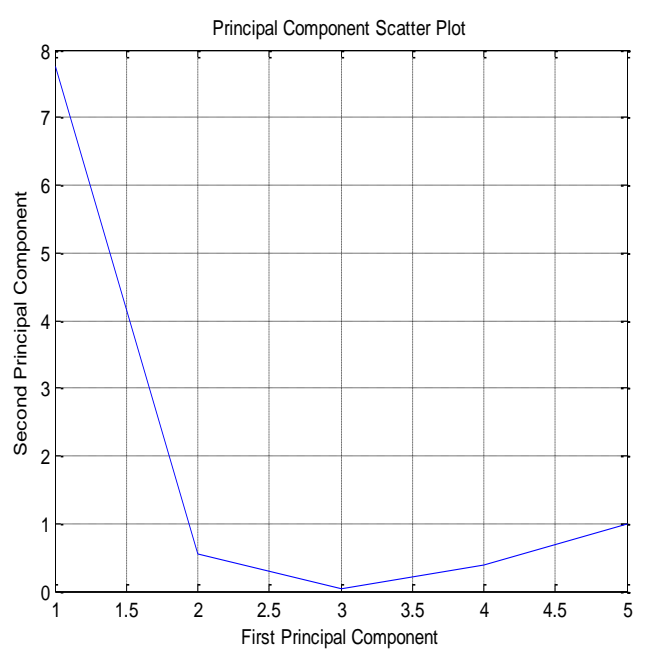

Fig. 4: GLCM feature model

\subsubsection{Correlation measure for subset selection}

Feature selection model not only selects the salient features that are relevant to the biometric class, also exploits the redundancy within the feature sets. Therefore, here with the context of feature selection from high dimensional data many redundant features may exist with pure relevancy to the class objects. Appearancebased texture features will explore some level of correlation between biometrics of individual classes which is referred to interuser similarity as shown in Fig. 5.

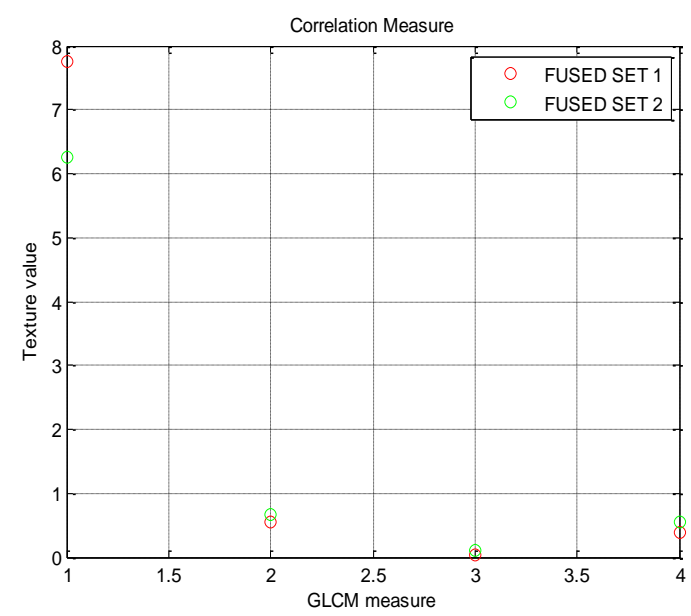

Fig. 5: Correlation among GLCM feature subsets

\subsection{Template level fusion}

In [15] general the feature level fusion methods consider simple concatenation of the templates obtained from the various modalities or combining the templates using a simple arithmetic operation by varying the overlapping factor up to $100 \%$ in multibiometric system templates of the user corresponds to the individual biometric sources. Here based on triangular norms feature level fusion is implemented as a series of binary fusions. In the tnorms operations generalized sum, max and min rules are included to meet the conjunction and disjunction operations from a common domain shown significant discrimination as shown in Fig. 6.

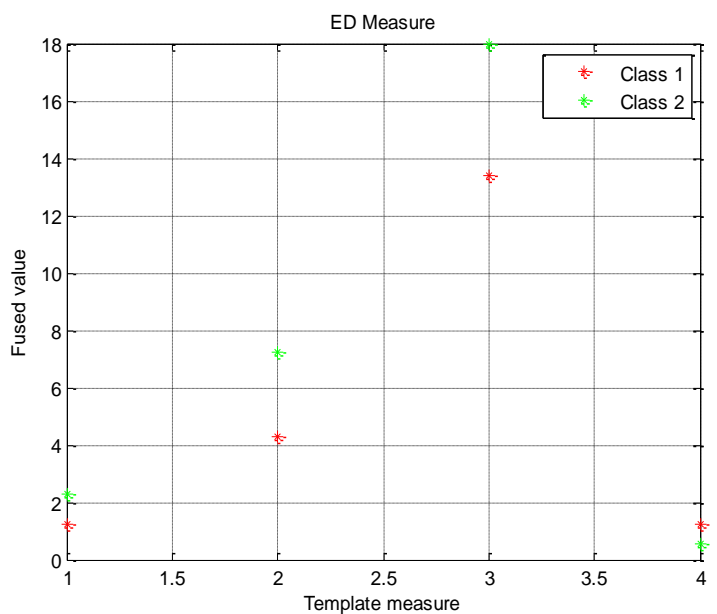

Fig. 6: Interclass Euclidean distance measure through template fusion model

\subsection{Biometric concealment}

Finally to solve security issues related to the template of the biometric system's user which is typically stored in a database simple permutation and modulo operation are carried out to encrypt the templates. Here both permutations and XOR operations are formulated based on the key input given as an encryption key. Each biometric identity is stored in transformed forms for future comparisons. It has the merits over conventional transformation models [13] since it is nearly impossible to recover the original template from the encrypted database and completely robust to any form of attacks.

\section{Experimental results}

Experiments are carried over on the publicly available iris, fingerprint and palm print biometric traits from biometric ideal test [14] 
using MATLAB software. Totally 1023 samples are collected from different users to formulate 223 multi-biometric classes to validate the performance metrics. Here we used simple Euclidean distance metrics driven KNN classifier to validate the performance metrics of the proposed multi-biometric system. In this technique, Hamming distance between the neighboring features is considered as the similarity measure to find the Nearest Neighbour (NN) from the template model stored in the database. To demonstrate the superiority of the proposed multi-biometric model, the performance measures such as False Acceptance Rate and False Rejection Rate are calculated. It is observed that the template fused biometric data outperforms the other models as it provides better matching with improved recognition accuracy as shown in Table 2.

Table 2: Classification performance analysis

\begin{tabular}{|c|c|c|c|}
\hline Encoding Methods & FAR (\%) & FRR (\%) & Accuracy (\%) \\
\hline Without PCA Transform & 20.2 & 18.8 & 80.16 \\
\hline PCA Transform & 15.16 & 5.34 & 85.45 \\
\hline Template Fusion & 7.34 & 3 & 97.89 \\
\hline
\end{tabular}

\section{Conclusions}

Here multi-biometric system focuses on recognizing the person by analyzing the texture patterns of four different biometric traits. In this work, we have developed a comprehensive statistical framework for signal level and feature level fusion in multi-biometric systems along with key driven template security. The wavelet multi-resolution approach provides optimal fusion performance and the composite images inherit the statistical parameters of input biometric images accurately. We investigated two different texture classification techniques for spatial patterns estimation, namely, binary pattern approach based on spatial relationship and a ternary pattern approach based on finite threshold driven texture classification models. We also proved that the multi-modal biometric system performance can be increased by employing the feature level fusion and appropriate feature selection methods. Finally, it is identified that the multi-biometric templates from different biometric sources such as fingerprint, multiple iris images and palm print modalities offers significant improvement in terms of classification rate.

\section{References}

[1] Kyong Chang, Kevin W. Bowyer, Sudeep Sarkar, Barnabas Victor, "Comparison and combination of ear and face images in appearance-based biometrics", IEEE Transactions on pattern analysis and machine intelligence, Vol.25, No.9, (2003), pp.1160-1165

[2] Anil K. Jain, Arun Ross, Salil Prabhakar, "An introduction to biometric recognition", IEEE Transactions on circuits and systems for video technology, Vol.14, No.1, (2004), pp. 4-20

[3] Zhijian Zhang, Rui Wang, Ke Pan, Stan Z. Li, Peiren Zhang, "Fusion of near infrared face and iris biometrics", International Conference on Biometrics, Springer, Berlin, Heidelberg, Vol.4642, (2007), pp. 172-180

[4] Yong Xu, David Zhang, Jing-Yu Yang, "A feature extraction method for use with bimodal biometrics", Pattern recognition, Vol. 43,No.3, (2010), pp. 1106-1115

[5] Abdenour Hadid, Jean-Luc Dugelay, Matti Pietikäinen, "On the use of dynamic features in face biometrics: recent advances and challenges", Signal, Image and Video Processing ,Vol.5,No.4, (2011), p. 495

[6] Raghavendra R., Bernadette Dorizzi, Ashok Rao, Hemantha Kumar G., "Designing efficient fusion schemes for multimodal biometric systems using face and palmprint", Pattern Recognition ,Vol.44,No.5 ,(2011),pp. 1076-1088

[7] Heng Fui Liau, Dino Isa, "Feature selection for support vector machine-based face-iris multimodal biometric system", Expert Systems with Applications, Vol.38,No.9, (2011),pp. 11105-11111

[8] Houda Benaliouche, Mohamed Touahria", Comparative study of multimodal biometric recognition by fusion of iris and fingerprint", The Scientific World Journal, Vol.2014, (2014), pp.1-13
[9] Lamis Ghoualmi, Salim Chikhi, Amer Draa,"A SIFT-based feature level fusion of iris and ear biometrics", IAPR Workshop on Multimodal Pattern Recognition of Social Signals in Human-Computer Interaction. Springer, Cham, Vol.8869,(2014),pp.102-112

[10] Ryan Connaughton, Kevin W. Bowyer, Patrick J. Flynn, "Fusion of face and iris biometrics", Handbook of Iris Recognition, Springer, London, (2013), pp. 219-237

[11] Haghighat Mohammad, Mohamed Abdel-Mottaleb, Wadee Alhalabi, "Discriminant correlation analysis: Real-time feature level fusion for multimodal biometric recognition", IEEE Transactions on Information Forensics and Security, Vol.11,No.9, (2016), pp. 1984 1996

[12] Kirti Gupta, Rashmi Gupta, "Multi-resolution wavelet-based image fusion for iris recognition", International Journal of Applied Pattern Recognition, Vol.2,No.2,(2015), pp. 182-198

[13] Devendra Reddy Rachapalli, Hemantha Kumar Kalluri, "A survey on biometrie template protection using cancelable biometric scheme", Electrical, Computer and Communication Technologies (ICECCT), 2017 Second International Conference on. IEEE, (2017), pp. 1-4

[14] http://biometrics.idealtest.org/index.jsp

[15] Alessandra Lumini, Loris Nanni, "Overview of the combination of biometric matchers" ,Information Fusion, Vol. 33, (2017),pp. 71-85 